\title{
EL CONFINAMIENTO COMO CONCEPTO CLAVE EN EL ESTUDIO DE LA INJUSTICIA SOCIAL HACIA LAS MUJERES TRANSGÉNERO*
}

\section{CONFINEMENT AS A KEY CONCEPT IN THE STUDY OF SOCIAL INJUSTICE TOWARDS TRANSGENDER WOMEN}

\author{
Jessica Tatiana Castaño-Urdinola**
}

\section{Resumen}

Objetivo. Presentar el discurso reflexivo sobre la justicia social que aporta Iris Marion Young, específicamente las cinco caras de la opresión, y cómo estas se evidencian en la vida de las mujeres trans que ejercen el trabajo sexual en Colombia. Metodología. Se basó en la revisión documental realizada para la construcción de los referentes conceptuales de la tesis de maestría en curso sobre injusticia espacial y resistencia en las mujeres trans trabajadoras sexuales de Manizales. Resultados. La explotación, la marginación, la violencia, el imperialismo cultural y la carencia de poder se presentan a diario en la cotidianidad de esta población. Conclusiones. Es necesario proponer el concepto del confinamiento como una sexta cara de la opresión para analizar las situaciones de injusticia social que enfrentan las mujeres transgénero, y la categoría de justicia espacial es clave para comprender la dimensión territorial de este tipo de fenómenos.

Palabras clave: justicia social, mujeres transgénero, opresión, confinamiento, justicia espacial.

\begin{abstract}
Objective. To present the thoughful discourse about social justice that Iris Marion Young provides, specifically the five faces of oppression, and how they are evident in the lives of trans-women who practice sex work in Colombia. Methodology. Based on the documentary review carried out for the construction of the conceptual referents requirements of an in progress Master's thesis about spatial injustice and resistance in the transgender women sex workers in de city of Manizales. Results. Exploitation, marginalization, violence, cultural imperialism, and lack of power are present in the daily life of this population. Conclusions. It is necessary to propose the concept of confinement as a sixth face of oppression to analyze the situations of social injustice faced by transgender women and the category of spatial justice is key to understand the territorial dimension of this type of phenomenon.
\end{abstract}

Key words: social justice, transgender women, oppression, confinement, spatial justice.

\footnotetext{
* Este artículo es resultado de la tesis en curso titulada: Prácticas colectivas de resistencia ante la injusticia espacial de las mujeres trans que ejercen el trabajo sexual en la Calle de las Guapas de Manizales, en el marco de la Maestría en Justicia Social y Construcción de Paz de la Universidad de Caldas.

** Universidad de Caldas. Manizales, Colombia. E-mail: jessica.castano@ucaldas.edu.co -

(D) orcid.org/0000-0001-7343-1483 Google Scholar
} 


\section{Introducción}

Existen distintas teorías de la justicia social en las que aparecen autores como Rawls, Sen, Nussbaum, Fraser, Young, entre otros. Cada uno se centra en uno u otro aspecto que considera vital para construir sociedades más justas socialmente, y es así como van a privilegiar la redistribución, las libertades, las capacidades, el reconocimiento, la participación o la eliminación de la opresión y la dominación como conceptos clave de sus teorías.

Este artículo emerge de la revisión documental realizada para la construcción de los referentes conceptuales de la tesis: Prácticas colectivas de resistencia ante la injusticia espacial de las mujeres trans que ejercen el trabajo sexual en la Calle de las Guapas de Manizales ${ }^{1}$, en el marco de la Maestría en Justicia Social y Construcción de Paz, y en él se presenta el discurso reflexivo sobre la justicia social de Young (2000), quien en su trabajo La justicia y la política de la diferencia, privilegia la opresión y la dominación como categorías que engloban las situaciones que viven los distintos grupos sociales.

Young propone cinco caras de la opresión que deberían reducirse o eliminarse para lograr la justicia social, que son: la explotación, la marginación, la carencia de poder, el imperialismo cultural y la violencia. Sin embargo, al abordar las realidades de las mujeres transgénero ${ }^{2}$ en Colombia, es necesario agregar una sexta cara de la opresión que dé cuenta de la dimensión espacial del fenómeno social de la injusticia, que hasta la década de los 80 -momento en que inicia el giro espacial en las ciencias sociales- había sido invisibilizada. Se concluye que el concepto de confinamiento adquiere gran relevancia en los estudios sobre las situaciones de injusticia social que atraviesan las mujeres trans, y que es teóricamente potente comenzar a integrar la justicia espacial como categoría de análisis.

\section{Referentes conceptuales}

Durante las últimas décadas se han presentado una serie de teorías sobre la justicia social que han enriquecido el panorama para comprender las distintas formas en que esta se expresa en

\footnotetext{
${ }^{1}$ En esta tesis de maestría se propone como objetivo general comprender la configuración de estas prácticas en ese espacio en el año 2018, y como objetivos específicos se plantean:

- Reconstruir el proceso de colectivización social de las Guapas en la ciudad de Manizales.

- Develar las consecuencias que produce la organización política del espacio para las trabajadoras sexuales trans de la Calle de las Guapas.

- Analizar los desacuerdos públicos que expresan las mujeres trans que ejercen el trabajo sexual en la Calle de las Guapas frente a las situaciones de injusticia que enfrentan.

${ }^{2}$ La Comisión Interamericana de Derechos Humanos (2015) define a las personas trans como: “Cuando la identidad de género de la persona no corresponde con el sexo asignado al nacer. Las personas trans construyen su identidad independientemente de tratamiento médico o intervenciones quirúrgicas" (p. 1). Esto quiere decir que las mujeres transgénero son aquellas que nacieron con una genitalidad interpretada como masculina, pero que a lo largo de su vida decidieron construir su identidad de género como mujeres.
} 
los individuos y los grupos sociales. Una de las perspectivas más sobresalientes es la de Rawls (1978), quien plantea la justicia como distribución, es decir, una sociedad justa es aquella en la que los bienes primarios - aquello que es necesario para satisfacer las necesidades- están distribuidos de forma adecuada. Los principios que orientarían esta distribución son:

- Justicia igualitaria: a cada persona una parte igual.

- Justicia según la necesidad: a cada persona de acuerdo con sus necesidades individuales.

- Justicia según el mérito: a cada persona según sus méritos.

- Principio de la diferencia: "Las desigualdades sólo se pueden justificar si benefician a los más desaventajados” (Murillo y Hernández, 2011, p. 13).

Esta visión de la justicia se orienta específicamente hacia los bienes que requieren los seres humanos, y a los cuales muchos no pueden acceder porque están mal distribuidos. Debe comprenderse a cada persona como un ciudadano libre e igual, por lo cual debe tener el acceso básico a libertades y derechos; debe poder desplazarse libremente y escoger su ocupación; debe tener la oportunidad de ocupar cargos en los espacios de toma de decisión; debe tener acceso a la riqueza y consolidar las bases sociales de respeto a sí mismo (Murillo y Hernández, 2011). Los bienes mencionados no son fines en sí mismos, sino medios que permiten construir sociedades más justas socialmente.

Sin embargo, otros autores de la justicia comienzan a criticar esta posición y es allí donde van a aparecer teóricos como Sen (1995), quien considera que la mirada de Rawls es instrumental. Para Sen, la justicia se refiere a las libertades que gozan las personas para poder elegir entre distintos modos de vivir, es decir, la capacidad de lograr realizaciones o lo que cada persona considera un fin. De acuerdo con Bedoya (2011), para Sen la justicia social debe ir de la mano del desarrollo humano, y ambos se logran cuando las personas pueden desarrollar y combinar sus capacidades con plena libertad, y esta última se garantiza generando oportunidades y concibiendo a las personas como agentes que toman sus propias decisiones.

Nussbaum $(2002,2007)$ complementa esta postura, agregando que las capacidades son lo esencial para una vida justa, y que el ordenamiento social (económico, jurídico y político) debe asegurar que las personas lleven vidas plenas a partir de la facilitación de estas capacidades. La autora presenta un sistema de 10 capacidades funcionales humanas centrales para la justicia, entre las cuales se encuentra la vida, la salud corporal, las emociones, la afiliación y el respeto por otras especies. Para Martínez (2015), Nussbaum plantea una teoría de la justicia social que puede servir de base para las políticas públicas y el derecho constitucional, y que incorpora la filosofía moral a la economía del desarrollo al momento de establecer lo que es la calidad de vida.

Por otro lado, Fraser (1997) pone sobre la mesa la discusión sobre dos formas de comprender la justicia social: distribución (dimensión económica) o reconocimiento (dimensión cultural- 
valorativa). Ella afirma que las luchas actuales por la justicia suelen enmarcarse en una u otra perspectiva, pero no buscan integrar ambas. A partir del análisis de las realidades que viven ciertos grupos, la autora propone que las injusticias son bidimensionales, es decir, se requiere una tercera vía en la que converjan ambas perspectivas, o como ella también lo llama: un marco integrador de los dos paradigmas.

Sin negar que existan otros autores que amplíen las perspectivas sobre la justicia social, se va a citar finalmente en este escrito a Young (2000), quien considera que el ejercicio del pensamiento sobre la justicia debería estar atravesado por dos conceptos clave: la dominación y la opresión, mas no por la distribución planteada por autores como Rawls como centro de la reflexión. Cabe mencionar que esta autora no pretende construir una teoría de la justicia, que estaría pensada con el fin de convertirse en un sistema normativo universal, sino más bien aportar un discurso reflexivo que esté situado histórica y contextualmente y que siempre esté “circunscrito a prácticas sociales y políticas concretas” (Young, 2000, p. 14).

La autora propone su forma de conceptualizar la opresión y la dominación: la primera se refiere a una serie de procesos institucionales que, al adquirir un carácter sistemático, se convierten en un obstáculo para el aprendizaje y uso de las habilidades de las personas en medios con reconocimiento social; también se relaciona con las limitaciones que estas tienen para comunicarse con otros y ser escuchadas. La dominación, por su parte, es definida como la imposibilidad o dificultad para que la gente participe en los espacios de toma de decisiones y de determinación de sus acciones, reforzada también por condiciones institucionales. Los movimientos sociales son una respuesta colectiva ante ambas situaciones y sus demandas dan cuenta de que si bien es necesario exigir la distribución de bienes materiales, sus luchas de fondo están orientadas a cuestiones estructurales, semánticas y relacionales que desbordan el paradigma distributivo.

Hasta aquí puede concluirse que, si bien existen múltiples perspectivas para explicar por qué en las sociedades actuales se configuran distintas situaciones de injusticia, es Young quien va a hacer uso de los conceptos de opresión y dominación para explicar por qué la diferencia representa una amenaza para el statu quo. Si bien las realidades de las mujeres transgénero pueden ser leídas desde cualquiera de los marcos explicativos presentados, a lo largo de este artículo se va a exponer de qué manera las cinco caras de la opresión dan forma de manera más concreta a sus injusticias, las cuales pueden complementarse con una sexta cara llamada "confinamiento".

\section{Metodología}

Estas reflexiones se producen a partir de la aplicación de la técnica de revisión documental, que permitió asumir una perspectiva teórica para el estudio de las situaciones de injusticia social que enfrentan las mujeres trans que ejercen el trabajo sexual en la "Calle de las Guapas" 
El confinamiento como concepto clave en el estudio de la injusticia social hacia las mujeres transgénero

de la ciudad de Manizales. En esta técnica el investigador "se dedica a reunir, seleccionar y analizar datos que están en forma de 'documentos' producidos por la sociedad para estudiar un fenómeno determinado” (Ferri, Muñoz, Ingelliz y Jabbas, 2005, p. 5), y también es conocida como indagación de fuentes secundarias.

Se revisaron distintos libros y artículos científicos que abordan la temática de la justicia social y se elaboraron Reseñas Analíticas Especializadas (RAES) para consignar los contenidos principales del texto. Finalmente, se codificaron, agruparon y analizaron las distintas perspectivas teóricas para elegir la más conveniente y cercana a la postura epistemológica del presente proyecto de investigación, que se ubica en la epistemología feminista.

Adicionalmente, los hallazgos conceptuales se contrastaron con algunos elementos que le han brindado la experiencia a la investigadora, por un lado, como integrante del semillero de investigación 'Pluriversos, Cultura y Poder', desde el cual se produce conocimiento alrededor de la acción colectiva y otros fenómenos sociales contemporáneos, y por el otro, como activista en 'Armario Abierto', organización autónoma, no gubernamental, privada y sin ánimo de lucro, desde la cual se diseñan estrategias que buscan aportar a la construcción de condiciones de existencia más justas para las personas que asumen identidades de género y/o ejercen sexualidades diversas.

A raíz de este trabajo, se han podido conocer de cerca las situaciones de injusticia social y espacial que atraviesan las mujeres trans que ejercen el trabajo sexual en la "Calle de las Guapas" de Manizales y compararlas con la situación de aquellas que habitan otras ciudades del país, y estos son insumos valiosos que han permitido confirmar la pertinencia de la perspectiva de la justicia que aquí se presenta y que se describe detalladamente en los resultados y la discusión.

\section{Resultados}

El concepto de opresión, que es central para el discurso de los grupos sociales que tanto le interesan a Young (2000), opera a través de cinco aspectos: la explotación, la marginación, la carencia de poder, el imperialismo cultural y la violencia. A continuación se explicará cada una de estas caras y se describirá cómo se presentan en las realidades de las mujeres transgénero colombianas.

La explotación es definida como "un proceso sostenido de transferencia de los resultados del trabajo de un grupo social en beneficio de otro" (Young, 2000, p. 88). Esta forma de opresión la viven aquellas personas que se ven obligadas a vender su fuerza de trabajo y que no logran ver transformadas sus condiciones de existencia, a pesar de la gran cantidad de tiempo que le dedican a su labor. Esta cara de la opresión también pasa por pensar quién hace ciertos trabajos, para quién y cómo se recompensa. Las mujeres transgénero acceden al trabajo sexual 
y al estilismo como únicas opciones laborales, de lo cual reciben una mínima cantidad que las subsume en la miseria. Muchas de ellas están formadas para hacer otro tipo de trabajos, pero los estereotipos que recaen sobre ellas no les permiten el acceso a esos cargos.

Cabe aclarar que aunque en ciudades como Bogotá, Medellín, Cali, entre otras, existen políticas públicas para los sectores LGBTI que buscan transformar las condiciones de existencia de esta población, según OutRight Action International (2016) el 79\% de las personas trans colombianas son discriminadas al momento de buscar un trabajo y solo el 5\% han conseguido firmar un contrato laboral; cuando lo consiguen, son obligadas a cambiar su vestuario y comportamiento. Esta organización reitera que gran parte de las mujeres trans acceden a trabajos transexualizados (estilismo y prostitución) por ser su única opción.

La marginación es para Young la forma más peligrosa de opresión. Las personas marginales son "aquellas a las que el sistema no puede o no quiere usar. (...) gente confinada permanentemente a una vida de marginación social, (...) que son expulsadas de una participación útil en la sociedad" (Young, 2000, p. 90). Esta cara de la opresión se expresa en múltiples privaciones materiales que son de carácter distributivo, pero también tienen una dimensión cultural, pues el ser marginal se convierte en un estatus; nadie desea ningún tipo de interacción con las personas marginales.

Las mujeres trans que ejercen el trabajo sexual se ven privadas del acceso a los bienes y servicios básicos de una sociedad como la salud, el trabajo, la educación, la seguridad y una sana alimentación. El solo hecho de pensar en las reacciones de los funcionarios de las instituciones al interactuar con ellas genera que construyan una barrera con el Estado; muchas veces las nombran como hombres y las juzgan por su identidad de género, lo que las expulsa de los espacios de reconocimiento social e institucional, por lo que ellas prefieren refugiarse en sitios periféricos. Según OutRight Action International (2016), en Colombia existe una jurisprudencia que "protege" a las personas trans, pero en las instituciones se antepone la subjetividad para aplicar la ley. Por ejemplo, aún las mujeres trans deben someterse a un proceso de patologización médica para poder acceder a intervenciones corporales. Se produce finalmente una triple discriminación que las ubica en lo más abyecto de lo marginal: son mujeres, son trabajadoras sexuales y son transgénero.

La carencia de poder, que es la tercera cara de la opresión, se refiere a "una posición en la división del trabajo y la posición social concomitante que deja a las personas pocas oportunidades para desarrollar y usar sus capacidades" (Young, 2000, p. 99). Sobre las mujeres transgénero se ejerce el poder, pero ellas no tienen posibilidad de ejercerlo, a excepción de las relaciones que se dan entre ellas. Las posibilidades que tienen de participar en procesos y espacios de toma de decisiones son mínimas, e incluso son otras personas que no hacen parte de sus comunidades las que toman decisiones sobre sus espacios de trabajo, horarios, formas de vestir y comportarse. La posibilidad, por ejemplo, de acceder a cargos públicos es limitada, por las situaciones explicadas 
anteriormente, pero ellas se han organizado y están resistiendo a esta situación. Según El Nuevo Día (2018), existe un aumento significativo en el número de candidatas transgénero en América Latina, pero solo Ecuador y Venezuela han logrado tener representantes trans en su Parlamento.

La cuarta cara es el imperialismo cultural, que se ha conceptualizado como la constante situación en la que los grupos sociales dominantes "proyectan sus experiencias como representativas de la humanidad. (...) El grupo dominante construye las diferencias que exhiben algunos grupos como carencia y negación. Estos son señalados como los otros" (Young, 2000, p. 103). En esta cara de la opresión los medios de comunicación juegan un rol crucial, y en el caso que se está analizando, las mujeres trans son víctimas de un régimen de visibilidad (Van Winkel, 2014) que se dedica a mostrar las imágenes que el público quiere ver: los cuerpos feminizados estereotípicos, las familias nucleares, los cuerpos como objeto de la publicidad, entre otros.

A este régimen le corresponde entonces su opuesto: un régimen de invisibilidad, que se dedica a ocultar los cuerpos "anormales", "indeseables” y "defectuosos". Las mujeres trans muy pocas veces son representadas mediáticamente desde lo que pueden llegar a ser educativa, laboral o artísticamente; son visibilizadas casi siempre como protagonistas de riñas callejeras, como personas que hurtan, como mujeres "indecentes" que venden sus cuerpos por dinero o como el típico personaje caricaturesco de telenovela que hace reír al público con sus comportamientos inusuales. Esto se explica, en parte, por la heteronormatividad que permea los patrones culturales en países como Colombia, entendida esta como la idealización de la heterosexualidad como única forma válida y posible de relacionamiento erótico afectivo (Warner, 1991). Así, cualquier expresión de la sexualidad que rompa este modelo se representa como anormal e inaceptable.

Por último, la violencia se define como una práctica social sistemática que "está dirigida a miembros de un grupo simplemente por ser miembros de ese grupo. (...) Es un hecho que todos saben que sucede y volverá a suceder” (Young, 2000, p. 108). Al respecto, los sectores poblacionales LGBTI son grupos altamente expuestos a la violencia en razón de sus identidades de género y orientaciones sexuales. Las mujeres trans que ejercen el trabajo sexual están en especial riesgo, pues son agredidas por los grupos armados, las fuerzas del Estado y la ciudadanía en general. Además las violencias no solo son físicas, sino también psicológicas y simbólicas, pues son agredidas verbalmente, tratadas como hombres, expulsadas de distintos espacios públicos y privados, rechazadas por sus familias, entre otras manifestaciones.

De acuerdo con los datos más recientes publicados por el Observatorio de Personas Trans Asesinadas $(2017)^{3}, 2.343$ personas de esta población han sido asesinadas en el mundo entre

\footnotetext{
${ }^{3}$ Este Observatorio inicia en Europa en el año 2009 como un proyecto de investigación que busca registrar, monitorear y analizar los reportes de las personas con orientaciones sexuales e identidades de género diversas asesinadas en todo el mundo. La información que recolectan se basa en las noticias que publican los distintos países vía internet, por lo tanto, faltan los datos que no se registran en estos medios o que no son denunciados.
} 
los años 2008-2016, siendo 1.834 de Latinoamérica y Centroamérica; el 64\% de ellas eran trabajadoras(es) sexuales. De acuerdo con la Comisión Interamericana de Derechos Humanos (2015), el 80\% de las mujeres transgénero de los países latinoamericanos mueren antes de los 35 años. Estas cifras dan cuenta de la cara de la violencia que atraviesa los cuerpos y las vidas de esta población.

A partir de lo expuesto, puede concluirse que el discurso reflexivo de la justicia propuesto porYoung, permite interpretar de forma concreta las situaciones de opresión que devienen en injusticia social para las mujeres trans, específicamente para aquellas que viven en condición de vulnerabilidad económica y social y que tienen como única posibilidad de supervivencia el trabajo sexual. A continuación se expondrá la pertinencia del involucramiento de una sexta cara de la opresión, que es el confinamiento espacial, para complementar esta perspectiva y poder leer de forma más precisa estas expresiones de la injusticia.

\section{Discusión}

A partir de lo explicado, se infiere que no es posible la justicia social si no se combaten las cinco caras de la opresión, algunas de las cuales tienen una dimensión distributiva, pero también se incluyen las del reconocimiento y la participación. Sin embargo, aunque las formas de opresión expuestas son claras y coherentes con la realidad de muchos grupos sociales, se considera desde esta investigación que hace falta explorar la dimensión espacial que tiene gran parte de los fenómenos de injusticia, y para explicarla vale la pena mencionar que desde finales del siglo $\mathrm{XX}$ se viene experimentando un giro espacial en las ciencias sociales y humanas, que da cuenta de una necesidad de contextualizar geográficamente los hechos en vista de la imposibilidad de universalizar las explicaciones de los fenómenos sociales. Los estudios culturales, territoriales y poscoloniales han contribuido especialmente a este giro.

La conceptualización de la justicia social no podía escapar a esta cuestión, y es por ello que varios autores han pensado cómo involucrar la dimensión espacial a la lectura de las injusticias. Uno de los intelectuales que asumió este desafío es Soja (2014), quien acuñó el concepto de justicia espacial, el cual se refiere a la organización política del espacio como un hecho social que puede ocasionar injusticias para ciertas personas o grupos. Este tipo de justicia, a diferencia de las definiciones revisadas de la justicia social, "busca privilegiar el espacio como categoría de análisis válida para interpretar tanto las condiciones que producen injusticias, como los conflictos que surgen por la búsqueda de una mayor justicia” (Soja, 2014, p. 81).

Así, desde esta conceptualización, el espacio no se considera neutral y únicamente físico, sino que se asume como un campo de lucha en el que se generan e impugnan injusticias físicas y simbólicas. Estas disputas no se reducen a las necesidades de redistribución, sino que también implica los conflictos sociales y culturales. Según Brennetot (2010): 


\begin{abstract}
(...) la dimensión espacial de la justicia involucra problemáticas muy diversas: la composición interna de los lugares, la distancia que los separa, los vínculos y las interfaces que los conectan, es decir, el conjunto de parámetros que contribuyen a la estructuración de los sistemas espaciales. Las relaciones entre los centros y sus periferias entran de este modo en la composición de la justicia espacial. (p. 80)
\end{abstract}

Puede inferirse que los estudios de la justicia espacial evidencian y se enfocan en la relación que existe entre el espacio y el poder, y uno de los autores que de mejor manera ha explicado este vínculo es Foucault (1999) con su noción de heterotopía, la cual se refiere a espacios físicos y mentales que cada sociedad construye y a los cuales se les asignan significados sobre lo deseable y lo indeseable. Si bien las heterotopías cuentan con varios principios, a continuación se explicará solo uno de ellos que, según Toro (2008), se refiere a la autorregulación, es decir, cualquier comportamiento que amenace los parámetros de normalidad debe ocultarse en espacios otros.

Para Foucault (1999), no existe sociedad alguna que no construya heterotopías, que en el caso de los encierros para los anormales van a recibir el nombre de heterotopías de crisis, que son lugares que alojan a sujetos que se desvían de la norma de comportamiento, sitios de encierro del anormal: clínicas psiquiátricas, prisiones y asilos de ancianos (García, 2006). Podría agregarse que algunas calles y plazas de las ciudades se han convertido también en espacios otros, que si bien no tienen barreras físicas que encierren a los individuos, sí configuran fronteras simbólicas que limitan la libre circulación de estos por otros espacios.

Volviendo a la relación mencionada entre espacio y poder, Foucault (1976) manifiesta que: "Podría escribirse toda una historia de los espacios - que sería a la vez una historia de los poderes-, desde las grandes estrategias de la geopolítica hasta las pequeñas tácticas del hábitat" (p. 192), lo que da cuenta de un vínculo que impacta cada espacio que se habita y que también incide en los significados atribuidos al tiempo, pues no será lo mismo el día, la tarde o la noche en determinados espacios. Occidente ha definido su propia espacialidad, distribuyendo social y políticamente los lugares, así como los atributos que se inscriben sobre ellos.

Se ha mostrado entonces que los asuntos espaciales tienen que ver directamente con la justicia y con el tratamiento que le brindan nuestras sociedades a la diferencia. Puede afirmarse, pues, que al integrar la categoría analítica de la justicia espacial al estudio de las realidades de los grupos sociales, en este caso de las mujeres trans, vale la pena integrar una sexta cara de la opresión frente a las cinco que propone Young, y esta es el confinamiento, que podría definirse, parafraseando a Inzulza y Galleguillos (2014), como la creación de espacios cerrados a partir de la transformación de espacios públicos, en los cuales se configura un clima de separación y desconfianza donde se estigmatiza la diferencia. Estos autores proponen que el confinamiento 
va de la mano de la seclusión socioespacial, que según Wacquant (2011) tiene que ver con el aislamiento de ciertos individuos y actividades sociales hacia determinados espacios.

Si bien Young propone la marginación como un proceso de exclusión, esa cara de la opresión tiene que ver con los estigmas sociales que se les asignan a ciertos grupos, es decir, como individuo no se interactúa con determinadas personas y se les hace a un lado por tener ' $\mathrm{X}$ ' o 'Y' características. Sin embargo, la cara del confinamiento tiene que ver directamente con el ejercicio de segregación de la diferencia a algún espacio que se vuelve periférico, no solo por su ubicación sino también por el sentido social y político que se le otorga. En el caso de las mujeres trans colombianas que ejercen el trabajo sexual, existen ciertos espacios de las ciudades en las que son confinadas y que dan cuenta de la necesidad de agregar esta cara a la conceptualización de la justicia social.

En Bogotá, por ejemplo, la zona de confinamiento para esta población se ubica entre las calles 19 y 24, con Avenida Caracas y carrera 18, específicamente en la localidad de Santa Fe. Allí son perseguidas y atacadas por miembros de la Policía Nacional y por grupos de exterminio o "limpieza social". Según lo investigado por Herrera, Lozano, Ortiz y Prada (2012): "En el barrio Santa Fe se encuentra ubicada la Zona de Tolerancia, establecida por la Alcaldía Mayor de Bogotá” (p. 162), y este espacio es concebido por las mujeres trans como su lugar seguro del cual prefieren no alejarse en su rutina diaria. Este espacio, para las autoras, se ha convertido en un gueto, pues “(...) por una parte, permite reglamentar el ejercicio de la prostitución, (...) al tiempo que crea barreras territoriales simbólicas que mantienen a las prostitutas lejos del resto de la ciudadanía, previniendo el deterioro moral de la sociedad" (p. 162). Este planteamiento guarda coherencia con lo que plantea Rosanvallon (2009), quien afirma que la sociedad actual está cada vez más fracturada y segregada, pues mucha gente pasa gran parte de su vida en guetos.

En Medellín, las zonas de seclusión socioespacial son el sector de Barbacoas en el centro de la ciudad y el Parque Lleras, en estos espacios las mujeres trans buscan clientes para producir recursos para la supervivencia, pero son continuamente expulsadas por la fuerza pública. Según lo publicado por Cervera (2013), existe un sector que se conoce popularmente como "La raza", y que ha sido cerrado con vallas por parte de la fuerza pública para impedir el acceso de los clientes a los bares y hoteles donde las mujeres trans ofrecen sus servicios. Ellas han tenido que desplazarse hacia otras zonas que ya están territorializadas por otras trabajadoras sexuales u otros actores, lo que las expone a disputas constantes por el espacio, especialmente con la Policía Nacional que, según ellas, abusa de la fuerza en los operativos.

En la calle 70 de Barranquilla existe una disputa constante entre mujeres transgénero y cisgénero que buscan controlar este espacio a través de lo que algunos vecinos del sector han llamado "la guerra del sexo". A la institucionalidad de la ciudad le llegan quejas todos los días, pues los habitantes del norte de Barranquilla rechazan la presencia de trabajadoras sexuales en 
El confinamiento como concepto clave en el estudio de la injusticia social hacia las mujeres transgénero

sus territorios. La Policía llega cada noche acudiendo al llamado de estas personas y realizan acciones conjuntas para desplazar a estas mujeres, sin brindarles otro tipo de posibilidades laborales. Pareciera que existe un interés mayor por "cuidar la imagen” de ciertos sectores de las ciudades y por erradicar aquello que incomoda a sus habitantes, que apoyar a quienes encuentran en el trabajo sexual su única posibilidad.

En Cali, por su parte, existen dos zonas donde las trans son confinadas: la carrera 15 con calle 98 y la calle octava con carrera sexta. Los habitantes de los barrios que se ubican en estas calles han interpuesto una tutela para que las trabajadoras sexuales trans sean reubicadas, pues argumentan que ellas son las protagonistas de constantes riñas, robos y escándalos. Estas expulsiones, tal y como en los casos anteriores, las obliga a buscar la territorialización de otros espacios que no siempre están disponibles, lo que las ubica en un estado de liminalidad forzada (Castillejo, 2000), es decir, no están ni aquí ni allá, ni adentro ni afuera, pues ningún lugar les es propio.

En Manizales el espacio de confinamiento socioespacial para estas mujeres está ubicado en el sector de la Galería, o Plaza de Mercado, a pocos metros del centro histórico de la ciudad. Según Castaño y Escobar (2000), la primera plaza de mercado de la ciudad estuvo ubicada en la actual Plaza de Bolívar entre 1849 y 1910. En ese último año se trasladó a la actual Plaza Alfonso López, y en el año 1950 se ubicó en el espacio actual que hace parte de la Comuna San José; en 1951 se inauguró oficialmente el pabellón central. Como en toda plaza de mercado, la dinámica principal de este espacio gira alrededor del comercio de distintos productos: frutas, verduras, plantas medicinales, herramientas, licores, entre otros, y también se ofrecen servicios sexuales a los que acceden hombres de distintos estratos socioeconómicos.

A una cuadra del pabellón central de la Plaza de Mercado se encuentra el espacio que actualmente se reconoce como "Calle de las Guapas", que se había popularizado hace años como "la Calle de la Penicilina”, y que por estar ligada a las dinámicas que caracterizan a las plazas de mercado, tales como "el ruido, los habitantes de calle, la acumulación de basuras y el consumo de sustancias psicoactivas, consolidó un imaginario social del lugar como 'peligroso', 'sucio' y 'desordenado'” (Castaño y Salazar, 2017, p. 209). La estética del lugar lo volvió oscuro e intransitable, pero aun así allí fueron llegando mujeres transgénero provenientes de distintos municipios del departamento de Caldas, cuya capital es Manizales, y de otras ciudades del país como Cali, Medellín, Barranquilla, Cartagena, entre otras, en búsqueda de cualquier rincón que pudieran territorializar para ofrecer servicios sexuales y así poder obtener recursos para la supervivencia diaria.

Con el paso del tiempo, y con mayor fuerza desde el año 2000 -según las anécdotas de las lideresas-, las trabajadoras sexuales transgénero fueron apropiándose cada día de esa calle, lo que no quiere decir que antes otras mujeres trans no ejercieran el trabajo sexual en otras zonas. 
Allí quedaron entonces estas mujeres, que decidieron hacer de esta calle su propio espacio y al cual comienzan a nombrar desde el año 2012 como "Calle de las Guapas”, ya que esta última palabra posee un significado que atraviesa sus vidas y se ha convertido en su lugar identitario. Cabe mencionar que gran parte de la población trans es fluctuante, pues precisamente por las situaciones de injusticia espacial que enfrentan, específicamente las agresiones policiales constantes y violencia por parte de sus clientes, deciden cambiar continuamente de ciudad en búsqueda de condiciones de existencia más justas.

Puede decirse, entonces, que el espacio juega un papel crucial en el sostenimiento de las desigualdades y las injusticias (Soja, 2014) y, en este caso, la "Calle de las Guapas” y los demás lugares de confinamiento de las ciudades citadas se configuran como espacios en los que quedan prácticamente encerradas las mujeres que los habitan y sobre los que recaen fuertes estigmas territoriales que terminan siendo asignados no solo a estos espacios sino también a sus ocupantes. En estos lugares de confinamiento aparecen fronteras invisibles que operan en doble vía: los habitantes de otras zonas de las ciudades temen transitar por estas zonas, especialmente en horas de la noche, pues se sienten en peligro, y las mujeres trans no pueden circular libremente por otros espacios porque la policía las violenta o la misma ciudadanía las discrimina de tal manera que ellas prefieren refugiarse en estos microespacios.

Cabe aclarar que aquí el confinamiento no se reduce a la delimitación espacial que se construye político-administrativamente para definir las zonas en las que puede ejercerse el trabajo sexual en las ciudades, sino que tiene que ver también con que las mujeres trans son desterradas de los demás espacios por los que pretenden circular, aun sin estar ejerciendo el trabajo sexual. En Manizales, por ejemplo, transitar por la Plaza de Bolívar se ha convertido en una odisea, pues la policía las expulsa continuamente argumentando que "dan una mala imagen de la ciudad". Adicionalmente, estas mujeres se sienten confinadas hasta en sus propios cuerpos, pues no pueden vestirse como ellas desearían y no pueden intervenir sus cuerpos sin atravesar un proceso de patologización médica y de autorización jurídica. Ellas viven en la liminalidad, pues no pueden estar "ni aqui”" "ni allá” por largas temporalidades.

Finalmente, se afirma que el confinamiento como sexta cara de la opresión se evidencia empíricamente en los ejemplos citados, cuyos espacios pueden ser leídos como guetos, que para Wacquant (2004) son:

(...) un instrumento socio-organizacional compuesto por cuatro elementos (el estigma, la restricción, el confinamiento espacial y el encasillamiento institucional), que emplea el espacio para reconciliar los dos propósitos antinómicos de la explotación y el ostracismo social. El gueto no es una "área natural" (...) sino una forma especial de violencia colectiva concretizada en el espacio urbano. (p. 72) 
El confinamiento como concepto clave en el estudio de la injusticia social hacia las mujeres transgénero

El confinamiento - o guetización - que sufren las mujeres trans profundiza las brechas existentes entre ellas y las demás personas, pues el hecho de excluirlas y encerrarlas en determinados espacios urbanos genera una mayor exotización de la diferencia y una justificación frente al aislamiento de lo supuestamente aberrante, muchas veces legitimado desde los discursos y prácticas institucionales. El confinamiento emerge, entonces, como un problema teórico y social relevante en el caso de las mujeres trans, lo que puede extrapolarse a otros fenómenos sociales que implican la innegable relación entre el espacio y el poder, tales como las realidades de comunidades étnicas, campesinas, refugiadas, entre otras. Las lecturas de la justicia social son más completas y precisas cuando involucran su dimensión espacial.

\section{Conclusiones}

Existe un amplio panorama de teorías sobre la justicia social, sin embargo, para efectos de la investigación que se está llevando a cabo, resulta pertinente la perspectiva deYoung, quien no pretende construir una teoría de aplicación universal, sino un discurso reflexivo sobre la justicia que sea contextual y localizado y que parta de las categorías de opresión y dominación, que en el fenómeno particular de interés están evidentemente manifiestas.

A partir de los resultados expuestos, puede concluirse que el confinamiento debe ser incluido como una sexta cara de la opresión - adicional a las propuestas por Young- en los estudios que abordan las injusticias sociales que enfrentan las mujeres transgénero, especialmente aquellas que ejercen el trabajo sexual en las ciudades colombianas y latinoamericanas. Si bien la autora plantea la cara de la marginación, esta posee un carácter social mas no específicamente espacial, y en los ejemplos presentados se evidencia que las mujeres trans no solo son estigmatizadas y excluidas en las interacciones sociales, sino que también son aisladas a espacios periféricos.

La producción de conocimiento sobre los fenómenos espaciales de confinamiento, seclusión o guetización se convierte en una posibilidad para llamar la atención, denunciar y visibilizar estas situaciones, para que puedan ser transformadas a través del quehacer de los colectivos sociales, los movimientos sociales y la institucionalidad. Estas disputas se juegan el derecho a la ciudad, que le está siendo negado a quienes rompen los modelos de normalidad sexual, social, de género, entre otros.

- Finalmente, se concluye que el involucramiento de la dimensión espacial a los fenómenos de injusticia social brinda posibilidades analíticas e interpretativas de gran riqueza, ya que toda situación se desarrolla en un contexto localizado, como en el caso de los campesinos, las personas afro o indígenas, entre otros. La categoría "justicia espacial" resulta más abarcadora y con mayores precisiones en coherencia con las experiencias de las y los excluidos. 


\section{Referencias}

Bedoya, C. (2011). Amartya Sen y el desarrollo humano. Revista Memorias, 12, 277-288.

Brennetot, A. (2010). Pour une géoéthique. Éléments pour une analyse des conceptions de la justice spatiale. L'Espace géographique, 1, 75-88.

Castaño, J. y Escobar, V. (2000). Direccionamiento estratégico del Centro Galerías Plaza de Mercado de la ciudad de Manizales, con fundamentación en la caracterización social y económica de sus locatarios y en el diagnóstico del comercio informal en su espacio público en el año 2000. Manizales, Colombia: Universidad Autónoma de Manizales.

Castaño, J. y Salazar, M. (2017). La Marcha de las Putas: sexualidad, control y resistencias. Cuestiones de género: de la igualdad y la diferencia, 12, 201-219.

Castillejo, A. (2000). Poética de lo Otro, para una antropología de la guerra, la soledad y el exilio interno en Colombia. Bogotá, D.C., Colombia: Universidad Nacional de Colombia.

Cervera, A. (2013). Transexuales, afectadas por intervención del Centro de Medellín. ElTiempo. Recuperado de http:/ /www.eltiempo.com/archivo/documento/CMS-12818382

Comisión Interamericana de Derechos Humanos. (2015). Violencia contra personas lesbianas, gay, bisexuales, trans e intersex en América. Washington, D.C.: Organización de los Estados Americanos.

El Nuevo Día. (2018). Candidatos transgénero ganan terreno en la política en América Latina. Recuperado de https://www.elnuevodia.com/noticias/mundo/nota/ candidatostransgenerogananterrenoenlapoliticaenamericalatina-2405212/

Ferri, J., Muñoz, A., Ingellis, A. y Jabbaz, M. (2005). Técnicas cualitativas de investigación social. Recuperado de http://ocw.uv.es/ciencias-sociales-y-juridicas/tecnicas-cualitativas-deinvestigacion-social/tema_6_investigacion_documental.pdf

Foucault, M. (1976). El espacio del poder. Recuperado de http://studylib.es/doc/5073760/ vii.-el-espacio-del-poder

Foucault, M. (1999). El lenguaje del espacio. Barcelona, España: Paidós.

Fraser, N. (1997). Iustitia interrupta: reflexiones críticas desde la posición "postsocialista”. Bogotá, D.C., Colombia: Siglo XXI - Uniandes.

García, M. (2006). Espacio y Poder: El espacio en la reflexión de Michel Foucault. Ciudad de México, México: Universidad Autónoma Metropolitana.

Herrera, S., Lozano, L., Ortiz, A. y Prada, N. (2012). ¡A mí me sacaron volada de allá! Relatos de vida de mujeres trans desplazadas forzosamente hacia Bogotá. Bogotá, D. C., Colombia: Alcaldía 
El confinamiento como concepto clave en el estudio de la injusticia social hacia las mujeres transgénero

Mayor de Bogotá, Universidad Nacional de Colombia y Asociación Colombiana para el Avance de la Ciencia.

Inzulza, J. y Galleguillos, X. (2014). Latino gentrificación y polarización: transformaciones socioespaciales en barrios pericentrales y periféricos de Santiago, Chile. Revista de Geografía Norte Grande, 58, 135-159.

Martínez, P. (2015). El "enfoque de las capacidades" de Martha Nussbaum frente el problema de la ética animal. Recuperado de http://www.scielo.cl/scielo.php?script=sci_arttext\&pid $=$ S0718-92732015000200004

Murillo, J. y Hernández, R. (2011). Hacia un concepto de Justicia Social. Revista Iberoamericana sobre Calidad, Eficacia y Cambio en Educación, 9 (4), 8-23.

Nussbaum, M. (2002). Las mujeres y el desarrollo humano. Madrid, España: Herder.

Nussbaum, M. (2007). Las fronteras de la Justicia. Madrid, España: Paidós.

Observatorio de Personas Trans Asesinadas. (2017). Transrespeto versus Transfobia. Recuperado de https:/ / transrespect.org/es/research/trans-murder-monitoring/

OutRight Action International. (2016). La situación de las personas trans en Colombia. Recuperado de https://www.outrightinternational.org/content/la-situaci\%C3\%B3n-de-laspersonas-trans-en-colombia

Rawls, J. (1978). Teoría de la Justicia. Ciudad de México, México: Fondo de Cultura Económica. Rosanvallon, P. (2009). La sociedad de los iguales. Madrid, España: RBA libros.

Sen, A. (1995). Nuevo examen a la desigualdad. Madrid, España: Alianza Editorial.

Soja, E. (2014). En busca de la justicia espacial. Madrid, España: Tirant Lo Blanch.

Toro, M. (2008). La heterotopía en Michel Foucault como concepto estético. Bogotá, D.C., Colombia: Universidad de La Salle.

Van Winkel, C. (2014). El régimen de la visibilidad. Criterios, 53, 14-22.

Wacquant, L. (2004). Las dos caras de un gueto. La construcción de un concepto sociológico. Renglones, 56, 72-80.

Wacquant, L. (2011). El diseño de la seclusión urbana en el siglo XXI. Herramientas, 48, 9-26.

Warner, M. (1991). Fear of a Queer Planet: Queer Politics and Social Theory. Minneapolis: University of Minnesota Press.

Young, I. (2000). La justicia y la política de la diferencia. Madrid, España: Ediciones Cátedra. 